\title{
A Fuzzy Trust Model for E-Commerce
}

\author{
Samia. Nefti, Farid Meziane and Khairudin Kasiran \\ School of Computing, Science and Engineering \\ University of Salford, \\ Salford, M5 4WT, UK
}

\begin{abstract}
:
Although, there are many successful E-commerce organizations, it is argued that E-commerce has not reached its full potential. Trust was often cited as the main reason as many customers are still skeptical about some online vendors. Many trust models have been developed, but most are subjective and did not take into account the vagueness and ambiguity of the domain and the specificity of customers. We have developed a model that attempts to identify the information customers expect to find on a vendors website to increase their trust and hence the likelihood of a transaction to take place. The system is supported by an information extraction system that facilitates the information gathering process. In this paper, we present a method based on fuzzy logic to evaluate trust in E-commerce based on the extracted information. We argue that fuzzy logic is suitable for trust evaluation as it takes into account the uncertainties within E-commerce data and like human relationships, trust is often expressed by linguistics terms rather then numerical values. The results of the system validation using two case studies are also presented.
\end{abstract}

\section{INTRODUCTION}

E-commerce is seen as an extension of mail and phone order transactions [10] and is gaining popularity. In Ecommerce, business transactions are no longer bound to physical existence, geographic boundaries, time differences or distance barriers [3]. Even though some research shows that E-commerce has managed to place itself in the society, there are many hindrance factors which cause E-commerce to fail to reach its full potential. Han and Noh [4] found that several critical failure factors of E-commerce need to be addressed seriously by the industry to ensure that E-commerce usage will continue to grow. The findings are mainly on the dissatisfaction of customers on the unstable Ecommerce systems, a low level of personal data security, inconvenience systems, disappointing purchases, unwillingness to provide personal details and mistrust of the technology $[2,8,11,12,15]$. Indeed, customers may doubt the quality of the goods as they may find it difficult to engage in a transaction without proper testing, seeing and touching the products.

As with any business transaction, there is always a risk that something will go wrong and there is no guarantee that all parties will act as expected. In E-commerce this risk is even higher as transactions are performed in the virtual world and customers get the product only after paying for it. To be successful, E-commerce requires mutual trust that needs to be established between all participants.

Although there are many successful E-commerce organisations, a day can hardly pass without us hearing about a negative story related to transactions made through the Web. This range from delays in deliveries, quality of the goods and fraud. Indeed, consumers' lost to Internet fraud has increase from US\$3.2 millions in 1999 [1] to more than US\$ 14.5 millions in 2002 [14] and this is increasing every year. This has affected consumers' trust towards online business. The question that many customers are asking is "who to trust in the cyber space?" and most importantly, how to quantify trust? Many variables should be considered when attempting to quantify or just trying to understand the trust relationship between the vendor and the customer.

From a technology perspective Jøsang [5] has identified that it is possible to develop a model for trust and that this model is a model for beliefs. In developing this model Jøsang has formulated a framework which he calls "subjective logic" which is an extension of standard logic and in part probability theory. It is the assessment mechanism that must be used to evaluate the probability associated to the information and the assessment will then be used to assist in the establishment of the requisite trust. Since the concept of trust is subjective, it creates a number of unique problems that obviates any clear mathematical result. Hence, fuzzy logic is currently being investigated as a possible best fit approach as it takes into account the uncertainties within E-commerce data and like human relationships, trust is often expressed by linguistics terms rather numerically.

In [10], Manchala proposes a model for the measurement of trust variables and the fuzzy verification of E-Commerce transactions. He highlights the fact that trust can be determined by evaluating the factors that influence it, namely risk. He defines cost of transaction, transaction history, customer loyalty, indemnity and spending patterns as the trust variables. Each variable is measured using semantic labels. His 
notation is focused on defining when two trust variables are related by an Electronic Commerce Trust Relationship (ECTR). Using this ECTR, a trust matrix is constructed between the two variables and a Trust Zone is established. He also describes a method for trust propagation and the construction of a single trust matrix between vendor and customer that governs the transaction. The problem with Manchala's model is that (1) it is unclear which variables should be used by default for the best results; (2) it is unclear if it is actually possible for a computer to automatically establish that two variables are related by an ECTR. In his definition, he mentions a semantic relationship between the variables, but neglects to mention how this fact will be specified to the computer so that evaluation can be automated and (3) it is unclear if ECTR merging will scale in the face of large trust matrices. These concerns are all related to the viability of implementing his model. These models do not support a theoretic approach to trust and they are not suitable for Ecommerce [17].

To overcome these drawbacks, in this paper we present a novel approach to quantify trust based on Fuzzy Logic. In the trust model we developed in [13] and summarised in section 1, (1) we believe that all variables identified are important when evaluating the vendor's trustworthiness making our system more complex and robust; (2) Because all the variable are related, the implementation of the system caused less problems as we do not have to specify the degree of the relationships between the various variable; (3) The scalability of the system is not an issue for our model, as all variables are used in the evaluation process. The other advantages of our system are its modularity, reusability and portability as described in section 3 .

The remaining of the paper is organised as follows: section 2 summarise the trust model developed and in section 3 we present the fuzzy inference system and in section 4 we explain how fuzzy logic can be used to evaluate and quantify the trust model. This is followed by the development of the rules base in section 5 . In section 6 we report the results of the application of the fuzzy system using two case studies. Finally, the conclusions and future development are presented in section 7 .

\section{THE TRUST MODEL}

In our trust model (Fig 1), we looked at the kind of information customers are looking for on a vendor's website to help them decide whether to engage in a transaction or not. The model identified four major factors that need to be looked at before dealing with unknown cyber merchants. These are: Existence, Affiliation, Policy and Fulfilment. Establishing the existence of merchants is an important factor in trusting them. Indeed the customer feels more comfortable when he knows that there is more then just an email behind a merchant's website. The information the customer needs to collect to satisfy the existence factor includes physical existence such as the merchant's Telephone Number, Fax and Address; mandatory registration and peoples' existence. The affiliation factor looks at third party endorsement, membership and portal [7]. The policy factor looks at information with regards to customer satisfaction policy, privacy statement and warranty policy. Finally, the fulfilment factor looks at delivery methods, methods of payment and the community comments. This is a large amount of information that needs to be collected and analysed and if done manually this process would be time consuming and may discourage many customers checking the information or performing a transaction with the merchant. In order to facilitate the process, we developed an information extraction system to support the data collection process [13].

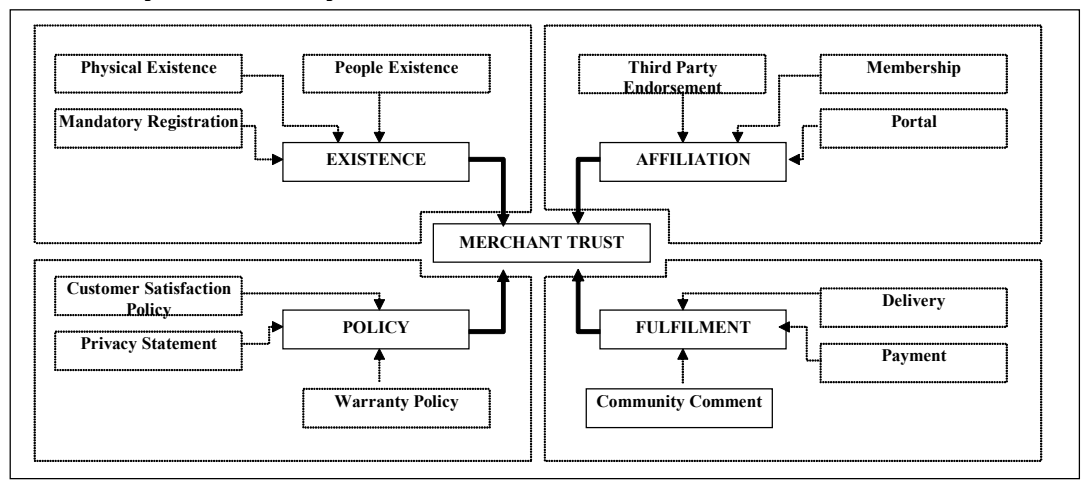

Fig. 1: The Trust Model 


\section{FUZZY INFERENCE SySTEM}

There are two concepts within fuzzy systems, which play a central role in our application purposes. The first one is a Linguistic variable, i.e. a variable whose values are words or sentences in a natural or synthetic language. Fuzzy set theory, which is based on such paradigm, deals with the ambiguity found in semantics [18]. The second concept is that of a fuzzy IF-THEN rule in which the antecedent and the consequent parts are propositions containing linguistic variables [9]. These two concepts are effectively used in the 'fuzzy logic controller paradigm' as shown in Fig. 2. The numerical values of the inputs $x_{i} \in U_{i} \quad(i=1, \ldots, n)$ are fuzzified into linguistic values, $F_{1}, F_{2}, \ldots, F_{n}$ where $F_{i}$ 's are defined as fuzzy sets in the input universe of discourse $U=U_{1} \times U_{2} \times \cdots \times U_{n} \subset \mathfrak{R}^{n}$. A fuzzy inference engine judges and evaluates several linguistic values $G^{l}, G^{2}, \ldots, G^{3}$, in the output universe of discourse $V$ by using fuzzy IF-THEN rules which are defined in the rule base;

$$
R^{(j)}: I F x_{i} \in F_{1}^{j} \text { and ...and } x_{n} \in F_{n}^{j} \text { THEN } y \in G^{j}
$$

where $(j=1, \ldots, M)$ and $M$ is the number of rules in the principle base. Each fuzzy IF-THEN rule in form of (1) defines a fuzzy set $F_{1}^{j} x F_{2}^{j} x \ldots x F_{n}^{j} \rightarrow G^{j}$ in the product space $U \times V$. Let $\mathrm{A}^{\prime}$ be an arbitrary input fuzzy set in $U$. A fuzzy set $B^{m}$ in $V$ can be calculated as:

$\mu_{A^{\prime} \circ R^{m}}(y)=\oplus\left(x_{i}, \ldots, x_{n}\right)^{T} \in U\left[\mu_{A^{\prime}}\left(x_{1}, \ldots, x_{n}\right) \otimes \mu_{F_{1}^{m} x_{,}, \ldots, F_{n}^{m} \rightarrow G_{n}^{m}}\left(x_{1}, \ldots, x_{n}, y\right)\right]$

where $t$-norm $\otimes$ and $s$-norm $\oplus$ are used for the intersection and the union operations respectively. The final output is a fuzzy set in $V$, which is a combination of the $M$ fuzzy sets, $A^{\prime} \circ\left(R^{(1)}, \ldots, R^{(m)}\right)$ The membership function of this inferred fuzzy set will be:

$$
\mu_{A^{\prime} \circ\left(R^{(1)}, \ldots \ldots, R^{(M)}\right)}(y)=\mu_{A^{\prime} \circ R^{(1)}}(v) \oplus \ldots \ldots \ldots \oplus \mu_{A^{\prime} \circ R^{(M)}}(y)
$$

The above membership function defines the fuzzy value of the output action $\mu_{B}(y)$. The crisp value of the output action can be obtained, say, by using the Centre of Gravity (COG) defuzzification method, where the shape of membership function, $\mu_{A^{\prime} \circ\left(R^{(1)}, \ldots \ldots, R^{(M)}\right)}(y)$, is considered to determine the crisp value of the output action $y^{\prime}$ where:

$$
y^{\prime}=\frac{\sum \mathrm{y} \mu_{B}}{\sum \mu_{B}}
$$

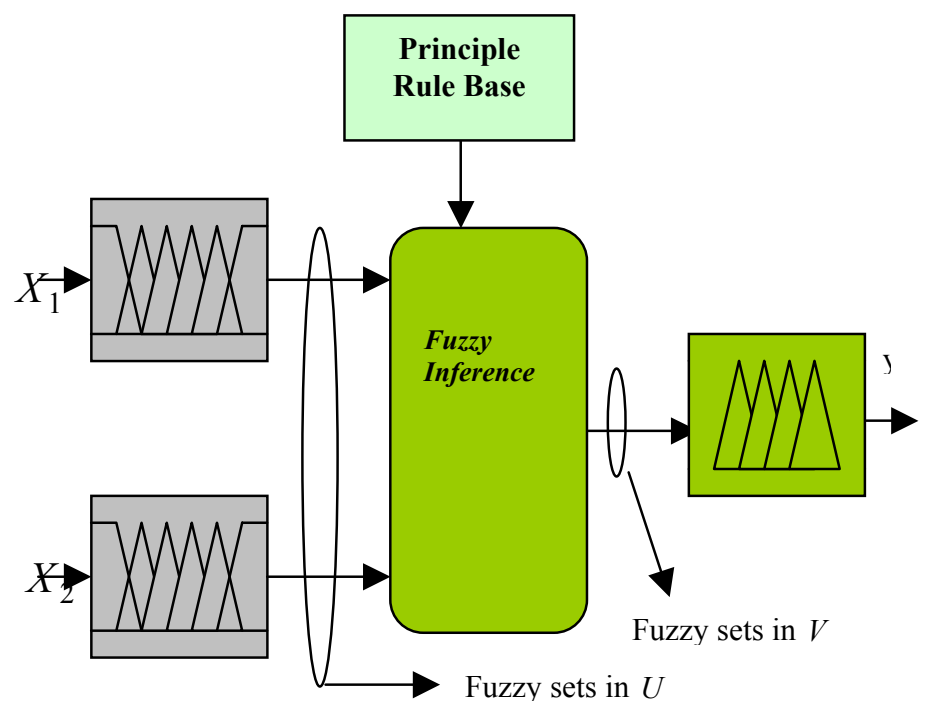

Fig. 2: The Fuzzy logic controller

\section{FUZZY LOGIC FOR TRUST MODEL}

The trust relationships among customers and vendors are hard to assess due to the uncertainties involved. Two advantages of using fuzzy-logic to quantify trust in E-commerce applications are: (1) Fuzzy inference is capable of quantifying imprecise data and quantifying uncertainty in measuring the trust index of the vendors. For example, in the trust model, the community comments variable in the fulfilment factor has a wide range of values as we may have a small or large number of customers providing positive or negative feedback to the vendor; the number of comments will affect the decision made by the associated evaluation module. (2). Fuzzy inference can deal with variable dependencies in the system by decoupling dependable variables. An example in our trust model is the mandatory registration variable in the existence factor that is dependent on the membership and third party endorsements variables in the affiliation factor. Indeed, if an organisation is a member of an association or endorsed by a third party, we assume that this organisation is fully registered with the required authorities even though the mandatory registration was not found and the value 0 was assigned by the information extraction system. Hence, the value of the output of module 2 will highly depend on the values of the third party endorsement and membership variables of the 
affiliation factor as these are judged to be more important to the customer then the portal and this output will be used as an input to the first module. This leads us to make a slight modification to the information model given in Fig. 1.

The general trust model proposed in this section is composed of five modules. Four modules will be used to quantify the trust measure of the four factors identified in our trust model (Existence, Affiliation, Policy and Fulfilment). The fifth module will be the final decision maker.

The inputs of the first module are the Existence variables which represent Physical Existence, People Existence and Mandatory registration and the output of the affiliation module (Fig. 3a). For module 2, the inputs are the Affiliation variables represented by Third Party Endorsement, Membership and Portal (Fig. 3b). Module 3 inputs are Policy variables and are represented by Customer Satisfaction, Privacy and Warranty (Fig. 3c). Finally, the fourth module has as inputs the Fulfillment variables and they are represented by Delivery, Payment Methods and Community Comment (Fig. 3d).

The Finale module is the decision maker as shown in Fig. 3e and will have as inputs the outputs of the four modules which are Existence_Index, Fulfilment_Index, Policy_Index and the Affiliation_Index. The output of this module is the trust index of the transaction. In our scheme, this trust index is determined by the success rate of all these variables.

In next section, we show a systematic method to produce the trusted conditions for vendors' websites. The trust index increases with the increase of all contributing factors and could decrease if not all these factors are satisfied. In Fig.3 (e), we plot the variation of the trust index of a resource site enhanced from low to high values

In the fuzzification phase, two membership functions described by the labels Small and High will be used for each variable related to each factor and the output of each module. For the decision maker module, we use three membership functions for the inputs and output. Fig. 4 shows the three membership functions corresponding to Low, Average and high degree of trustworthiness. They map a variable $x$ into the interval $[0,1]$, with 1 for full trust and 0 for no trust. The membership functions are represented by Gaussians defined by the parameters $(m, \sigma)$ where $m$ is the center and $\sigma$ the standard deviation. For example a vendor's website with 0.75 trust index is considered high and should be trusted.

The inference rules are subject to designer's choice based on criteria of the risk and the gain as defined by Tao and Thoen [16]. Fuzzy inference is a process to assess the trust index in five steps: (1) Register the initial values of each variable as defined by the information extraction system. (2) Use the membership functions to generate membership degrees for each variable related to each module. (3) Apply the fuzzy rule set defined in each module onto the output space (trust index) through fuzzy 'and' and 'or' operations. (4) Aggregate the outputs from each rules related to each module, and (5) derive the factor index through a defuzzification process using the centroid method. These same steps will also be used for the fifth module to generate the trust index.

\section{THE CONSTRUCTION OF THE RULES BASE}

The decision to trust or not to trust E-commerce as a shopping medium is up to consumers' evaluation, and their evaluation can be based on many factors such as price, convenience, selection of choice and the information available on the merchant's website like those defined in our model. It is widely accepted that if the economic gain is greater than the risk involved then the transaction is reasonably viable. Based on this assumption, Tao and Thoen [16] formalised the process as follows:

$$
\mathrm{G}_{\mathrm{b}}=\mathrm{P}_{\mathrm{b}} \mathrm{L}_{\mathrm{b}}
$$

where $G_{b}$ is the gain entering the E-commerce transaction, $\mathrm{P}_{\mathrm{b}}$ is the risk that the consumer takes for trusting the E-commerce merchants and $\mathrm{L}_{b}$ is the loss the consumer has to bear when the transaction does not produce the result as expected. Consumers are usually proceeding with the transaction if the potential gain is greater than the potential lost and will be indifferent if both values are equal. Thus one has either to maximise the gain $G_{b}$ or minimise the risk $\mathrm{P}_{\mathrm{b}}$. The risk can be minimised by providing all the information required by the customer on the vendor's website. Based on this model, we assume that if a large amount of information is available on a vendor's website and if this information is valid then the vendor can be trusted. In addition, some information is more important then others. For example, in the affiliation factors, third party endorsement and membership have more weight then portal. Similarly, community comments might be more important then payment and delivery methods. Therefore, some inputs will have more weight then others. Examples of rules used in Module1 are: 
1. If (Physical_existence is Low) and (people_existence is Low) and (Mandatory registration is Low) and (output2 is Low) then (existence_index is Low)

2. If (Physical_existence is Low) and (people_existence is Low) and (Mandatory_registration is High) and (output2 is High) then (existence_index is High)

3. If (Physical_existence is Low) and (people_existence is High) and (Mandatory_registration is Low) and (output2 is Low) then (existence_index is Low)

4. If (Physical existence is Low) and (people existence is High) and (Mandatory_registration is Low) and (output2 is High) then (existence_index is Average)

5. If (Physical_existence is Low) and (people_existence is High) and (Mandatory_registration is High) and (output2 is High) then (existence_index is High)

Examples of the rules used in Module5 are:

1. If (Existence_Index is Low) and (Affiliation_Index is Low) and (Policy Index is Low) and

(Fulfillment_Index is Low) then (Trust-Index is Small)

2. If (Existence_Index is Low) and (Affiliation_Index is Medium) and (Policy Index is Low) and Fulfillment_Index is Meduim) then (Trust-Index is Average)

3. If (Existence_Index is Low) and (Affiliation_Index is Medium) and (Policy_Index is Low) and Fulfillment_Index is High) then (Trust-Index is Average)

4. If (Existence_Index is Average) and (Affiliation_Index is High) and (Policy_Index is high) and (Fulfillment_Index is High) then (Trust-Index is Big)

5. If (Existence Index is High) and (Affiliation Index is Low) and (Policy_Index is Low) and (Fulfillment_Index is High) then (Trust-Index is Average)

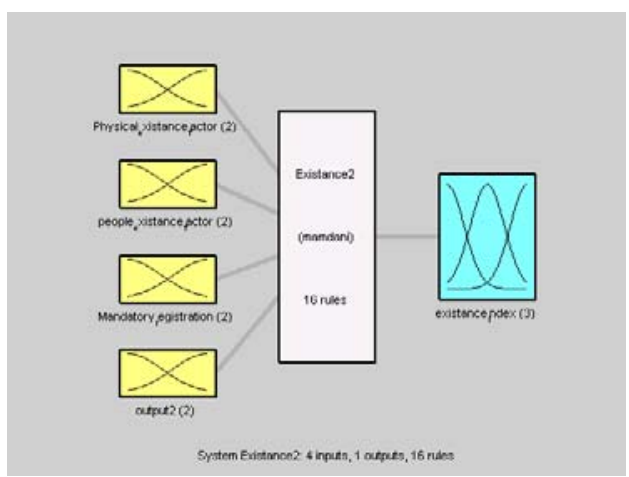

Fig. 3a: The existence module

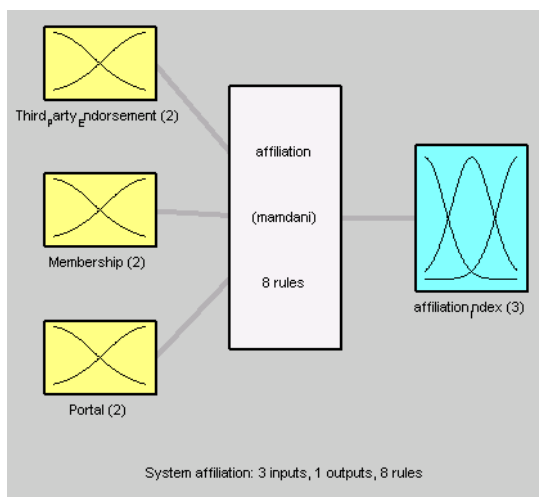

Fig. 3b: The Affiliation module

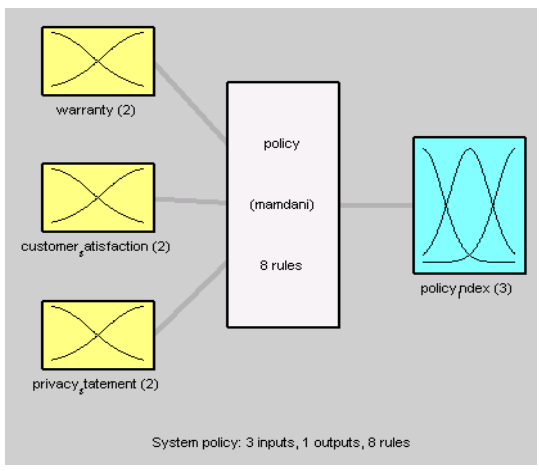

Fig. 3c: The Policy module 


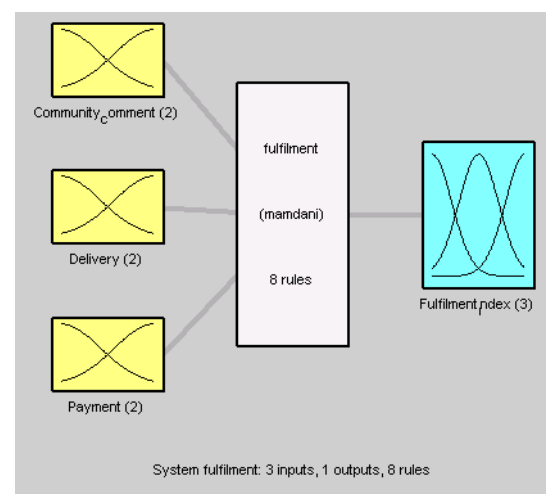

Fig. 3d: The Fulfilment module

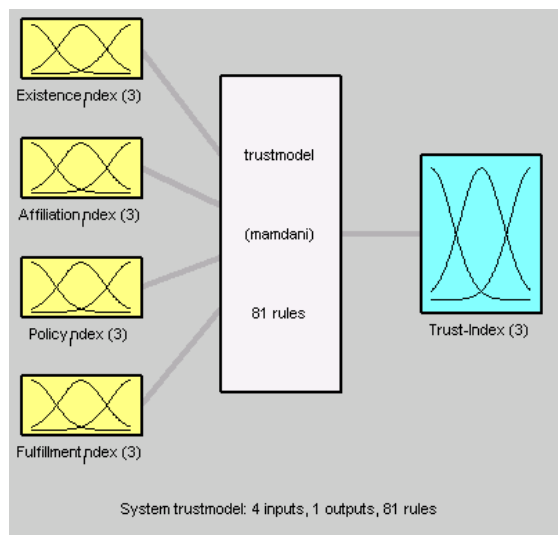

Fig. 3e: The Final decision maker module

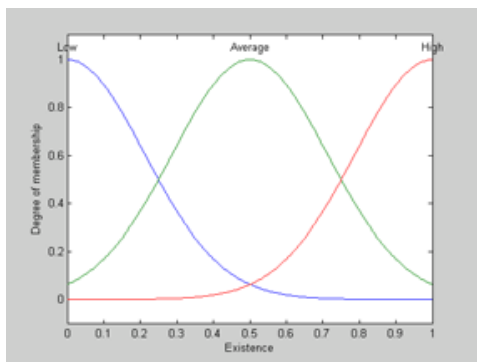

Fig. 4: Membership function for the trust index

\section{CASE STUDIES}

To illustrate the model developed in this paper, we consider two scenarios. The first scenario is Denimjunkies $^{1}$, a vintage clothing shop selling used collectable items such as jeans, jackets and shirts and the second scenario is Mesh Computers ${ }^{2}$, a company

\footnotetext{
${ }^{1} \mathrm{http}: / /$ denimjunkies.com/

${ }^{2} \mathrm{http}: / /$ www.meshcomputers.com
}

selling PCs and peripherals. The 4 factors of the trust model will be investigated. For each factor, a set of three variables will be considered hence, a total of 12 variables. Thus, in total combining all variables, results in a full factorial design of $2^{12}$.yielding to a total of 4096 possible combinations for each product. We use only two membership functions to describe each variable for each factor. Given the complexity of the problem, it becomes apparent why we grouped our variables into four factors which are processed by separate modules as defined earlier. This allows us to consider only 8 possible combinations per module. For the final decision support module, for more precisions, three membership functions are used low, average and high and this gives a possible total of 81 combinations for the final decision module. Table 1 summarizes the information extracted from the two case studies.

Table 1: the extracted information for the case studies ${ }^{3}$

\begin{tabular}{|c|c|c|c|c|}
\hline & & Variables & Scenario 1 & Scenario 2 \\
\hline \multirow{3}{*}{ 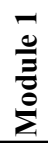 } & \multirow{3}{*}{ 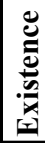 } & Physical Existence & $\mathrm{P} / \mathrm{A}$ & $\mathrm{P} / \mathrm{F} / \mathrm{A}$ \\
\hline & & People Existence & no & yes \\
\hline & & Registration & no & yes \\
\hline \multirow{3}{*}{ 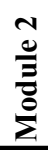 } & \multirow{3}{*}{ 离 } & Endorsement & no & yes \\
\hline & & Membership & yes & yes \\
\hline & & Portal & no & yes \\
\hline \multirow{3}{*}{ 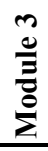 } & \multirow{3}{*}{$\begin{array}{l}\overrightarrow{0} \\
\frac{0}{0} \\
0\end{array}$} & Customer Satisfaction & yes & yes \\
\hline & & Privacy Statement & yes & no \\
\hline & & Warranty Policy & no & 2 years \\
\hline \multirow{3}{*}{ 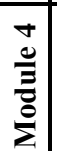 } & \multirow{3}{*}{ 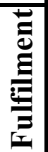 } & Delivery & immediately & delayed \\
\hline & & Payment & $\mathrm{Cr} / \mathrm{D} / \mathrm{Cq}$ & $\mathrm{Cr} / \mathrm{D} / \mathrm{Cq}$ \\
\hline & & Community Comments & no & no \\
\hline
\end{tabular}

Table 2 shows the fuzzification of the extracted values related to each variable. The output of the module 2 is also used as an input for module 1 . The following are some interpretations of the results. In the first scenario, the information extraction system found two sub-variables (phone number and address) out of a possible three; hence a value of 0.6 is assigned to the physical existence variable, thus after the fuzzification step the degree of membership function of this value is 0.4 for Small and 0.6 for High. The remaining two variables (people existence and mandatory registration) in the existence factor were assigned the membership function 0 (or small) as no information is found to represent them. In the

${ }^{3}$ P: Phone number; A: Address; F: Fax number; Cr: Credit card; D: Debit card; Cq: Cheque. 
second scenario, all information related to the existence factor was found; thus the degree of membership function of all three existence variables will be 1 .

Initially, in the fuzzification step the membership is determined by assessing all terms in the premise. After all selected rules are inferred in parallel. The fuzzy operator 'and' is applied to determine the support degree of the rules. The 'and' results are aggregated together. The final trust index for the scenario 1 is generated by defuzzifying the aggregation using centroid method.

Table 2: the fuzzification of the extracted information for the case studies

\begin{tabular}{|c|c|c|c|c|}
\hline & & Variables & Scenario 1 & Scenario 2 \\
\hline \multirow{5}{*}{$\begin{array}{l}\bar{\varrho} \\
\bar{\Xi} \\
\stackrel{\Xi}{\Xi} \\
\Sigma\end{array}$} & \multirow{5}{*}{ 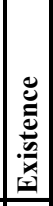 } & Physical Existence & $\{0.4 / \mathrm{S} ; 0.6 / \mathrm{H}\}$ & $\mathrm{H}$ \\
\hline & & People Existence & $\mathrm{S}$ & $\mathrm{H}$ \\
\hline & & Registration & $\mathrm{S}$ & $\mathrm{H}$ \\
\hline & & Output 2 & A & $\mathrm{H}$ \\
\hline & & Output 1 & A & $\mathrm{H}$ \\
\hline \multirow{4}{*}{ 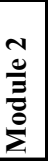 } & \multirow{4}{*}{ 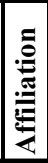 } & Endorsement & $\mathrm{S}$ & $\mathrm{H}$ \\
\hline & & Membership & $\mathrm{H}$ & $\mathrm{H}$ \\
\hline & & Portal & $\mathrm{S}$ & $\mathrm{H}$ \\
\hline & & Output 2 & A & $\mathrm{H}$ \\
\hline \multirow{4}{*}{ 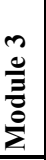 } & \multirow{4}{*}{ 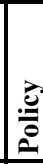 } & Customer Satisfaction & $\{0.8 / \mathrm{S} ; 0.2 / \mathrm{H}\}$ & $\mathrm{H}$ \\
\hline & & Privacy Statement & $\mathrm{H}$ & $\mathrm{L}$ \\
\hline & & \begin{tabular}{|l|} 
Warranty Policy \\
\end{tabular} & $\mathrm{S}$ & $\mathrm{H}$ \\
\hline & & Output 3 & $\mathrm{~S}$ & A \\
\hline \multirow{4}{*}{ 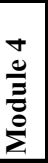 } & \multirow{4}{*}{ 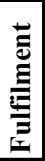 } & Delivery & $\mathrm{H}$ & $\mathrm{H}$ \\
\hline & & Payment & $\mathrm{H}$ & $\mathrm{H}$ \\
\hline & & Community Comments & $\mathrm{L}$ & L \\
\hline & & Output 3 & A & A \\
\hline
\end{tabular}

Example of the rules used to process the final decision for the scenariol are:

$\mathrm{R}_{1}$ : IF the Existence_Index is Average and Affiliation Index is Average and Policy_Index is Small and Fulfilment_Index is Small THEN the Trust Index is Small.

$\mathrm{R}_{2}$ : IF the Existence_Index is Small and Affiliation_Index is Small and Policy_Index is Small and Fulfilment_Index is Small THEN the Trust Index is Small.

There are many other fuzzy inference rules that can be designed using various conditional combinations of the fuzzy variables. The final trust index for the scenario 2 is 0.95 is generated by defuzzifying the aggregation.
Example of the rule used to process the final decision for scenario 2 is:

IF Existence is High and Affiliation is High and Fulfilment is Average and Policy is Average THEN the Trust Index is High.

As we can see from Fig. 5, the variable index increases with the increase of the contributing attributes of each variable with the highest weigh such as (registration and output 2 in the case of the first module), Endorsement and Membership in case of module 2 and community comments for module4. From Fig. 6 we can see that the output index of module 5 increases with the increase of the contributing attribute of the Affiliation and fulfillment Index. The variable index decrease when all attribute are not satisfied.

The final decision for scenariol is:

$\mathrm{R}_{11}$ : If the Existence is Average and Affiliation is Average and Policy is Small and Fulfilment is Small then the Trust Index is Low.

The final decision for the For scenario1 is :

$\mathrm{R}_{81}$ : If Existence is High and Affiliation is High and Fulfilment is Average and Policy is Average then the Trust Index is High.

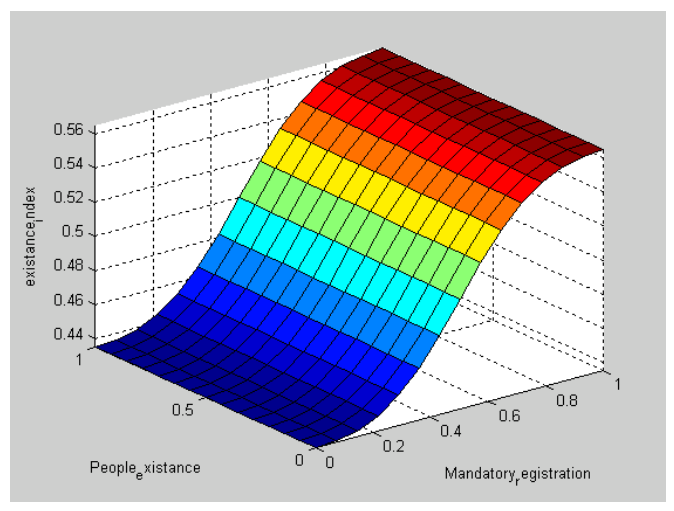

Figure 5 output of the Existence Module1 


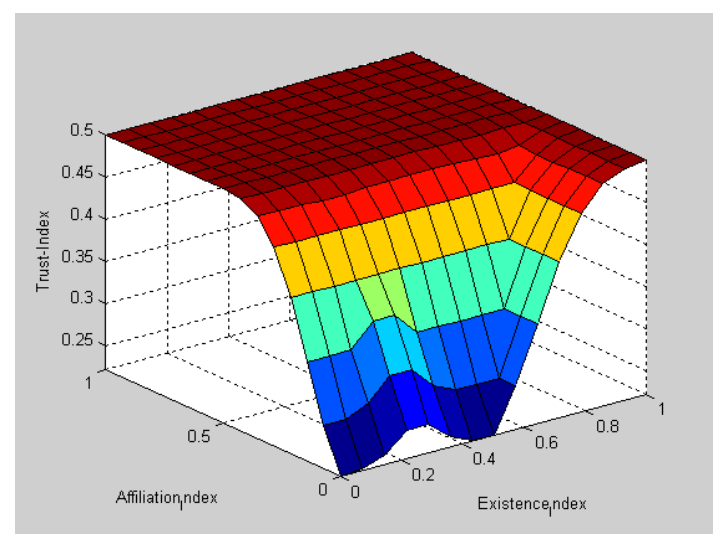

Fig. 6: Output of the finale decision maker Module

\section{CONCLUSION AND FUTURE WORK}

In this paper, we presented a system based on fuzzy logic to support the evaluation and the quantification of trust in E-commerce. Although, the system has addressed many issues that other systems did not such as taking into account the fuzzy nature of trust and using a substantial number of variables, we believe that the system can be improved in many ways. As stated in many trust models, there are other aspects that contribute to the completion of online transactions. This include the price, the rarity of the item and the experience of the customer In order to develop an effective decision support system, future development should include some if not all of these aspects. The price of the item is certainly an important variable as it is shown in many studies that if the price is reasonably low, customers are ready to take the highest risk to purchase the item. Online transactions also depend on customer's experience and personality. Some customers may value some variables more then others. Hence we believe that future systems should allow customers to rank trust variables according to their own perception and experience.

\section{REFERENCES}

[1] S. Ba, "Establishing Online Trust Through a Community Responsibility System", Decision Support Systems, (13):323336, 2001.

[2] Cheskin Research group, "eCommerce Study Trust", http://www.studioarchetype.com/cheskin/assets/images/etrust .pdf, 1999.

[3] J. Guo and C. Sun, "Global Electronic Markets and Global Traditional Markets" in:Wigand, Rolf T.; Kramer, Kenneth; Schmid (Editor), Beat F.; Pavlikova, Lucia:
Globalization and Electronic Commerce. - Electronic Markets, Vol. 14, No. 1, 2004.

[4] K.S. Han and M.H. Noh, "Critical Failure Factors that Discourage the Growth of Electronic Commerce", International Journal of Electronic Commerce. 4(2), pp. 2543, 1999.

[5] A. Jøsang, "Modelling Trust in Information Society", Ph.D. Thesis, Department of Telematics, Norwegian University of Science and Technology, Trondheim, Norway, 1998.

[6] M.K Kasiran. and F. Meziane, "An Information Framework for a Merchant Trust Agent in Electronic Commerce", In Hujan Yin, Nigel Allinson, Richard Freeman, John Keane and Simon Hubbard (Eds), Intelligent Data Engineering and Automated Learning, LNCS 2412, pp 243 248, Springer, 2002.

[7] M.K Kasiran and F Meziane, "The Usage of Third Party Endorsement in Ecommerce Websites", $7^{\text {th }}$ International Conference on Work with Computing Systems (WWCS2004), Kuala Lumpur, Malaysia, pp. 794-798, 2004.

[8] R. J. Lewicki, and B.B. Bunker, "Developing and Maintaining Trust in Working Relationships", In R. M. Kramer \& T. Tyler (Eds.), Trust in organizations. Thousand Oaks, CA: Sage, pp. 114-139, 1996

[9] E. M. Mamdani, "Application of fuzzy algorithms for control of simple dynamic plant,", IEE Proc. Control Theory Appl., vol. 121, no. 12, pp. 1585-1588, 1974. .

[10] D.W. Manchala, "E-Commerce Trust Metrics and Models", IEEE Internet Computing, March-April 2000, pp.36-44

[11] K.O. Matthew and E. Turban, "A Trust Model for Consumer Internet Shopping", International Journal of Electronic Commerce, 6(1):75-91, 2001.

[12] R.C. Mayer, J.H. Davis and F.D. Schoorman, "An Integrative Model of Organizational Trust", Academy of Management Review, 20(3):709-734, 1995.

[13] F. Meziane and M.K Kasiran, "Extracting Unstructured Information From the WWW to Support Merchant Existence in E-Commerce", in Antje Dusterhoft and Bernhard Thalheim (Eds), Lecture Notes in Informatics, Natural Language Processing and Information Systems, pp.175-185, GI-Edition, Bonn 2003, Germany.

[14] National Fraud Information Centre http://www.fraud.org/2002intstats.htm

[15] D. Shapiro, B.H. Sheppard, and L. Cheraskin, "Business on a Handshake", The Negotiation Journal, pages 365-378, October 1992.

[16] T. Tao-Huan and. W. Theon, "Towards a Generic Model of Trust in Electronic Commerce", International Journal of Electronic Commerce, 5(2):61-74, 2001.

[17] Tyrone W. A. Grandison. Trust Management for Internet Applications, PhD Thesis, Imperial College of Science, Technology and Medicine University of London, Department of Computing,2003

[18] L. Zadeh. "Fuzzy sets", Journal of Information and Control, 8:338--353, 1965 